\title{
On the age of Galactic bulge microlensed dwarf and subgiant stars ${ }^{\star}$
}

\author{
G. Valle $\mathrm{e}^{1,2,3}$, M. Dell'Omodarme ${ }^{3}$, P. G. Prada Moroni ${ }^{2,3}$, and S. Degl'Innocenti ${ }^{2,3}$ \\ ${ }^{1}$ INAF - Osservatorio Astronomico di Collurania, via Maggini, 64100 Teramo, Italy \\ e-mail: valle@df.unipi.it \\ 2 INFN, Sezione di Pisa, Largo Pontecorvo 3, 56127 Pisa, Italy \\ 3 Dipartimento di Fisica "Enrico Fermi", Università di Pisa, Largo Pontecorvo 3, 56127 Pisa, Italy
}

Received 5 February 2015 / Accepted 3 March 2015

\begin{abstract}
Context. Recent results by Bensby and collaborators on the ages of microlensed dwarf and subgiant stars in the Galactic bulge have challenged the picture of an exclusively old stellar population, because ages significantly younger than 9 Gyr have been found.

Aims. However, these age estimates have not been independently confirmed with different techniques and theoretical stellar models. One of the aims of this paper is to verify these results by means of a grid-based method. We also quantify the systematic biases that might be induced by some assumptions adopted to compute stellar models. In particular, we explore the impact of increasing the initial helium abundance, neglecting the element microscopic diffusion, and changing the mixing-length calibration in theoretical stellar track computations.

Methods. We adopt the SCEPtER pipeline with a newly computed stellar model grid for metallicities $[\mathrm{Fe} / \mathrm{H}]$ from $-2.00 \mathrm{dex}$ to $0.55 \mathrm{dex}$, and masses in the range $[0.60 ; 1.60] M_{\odot}$ from the zero-age main sequence to the helium flash at the red giant branch tip. By means of Monte Carlo simulations we show for the considered evolutionary phases that our technique provides unbiased age estimates.

Results. Our age results are in good agreement with Bensby and collaborators findings and show 16 stars younger than 5 Gyr and 28 younger than $9 \mathrm{Gyr}$ over a sample of 58 . The effect of a helium enhancement as large as $\Delta Y / \Delta Z=5$ is quite modest, resulting in a mean age increase of metal rich stars of $0.6 \mathrm{Gyr}$. Even simultaneously adopting a high helium content and the upper values of age estimates, there is evidence of 4 stars younger than $5 \mathrm{Gyr}$ and 15 younger than 9 Gyr. For stars younger than 5 Gyr, the use of stellar models computed by neglecting microscopic diffusion or by assuming a super-solar mixing-length value leads to a mean increase in the age estimates of about $0.4 \mathrm{Gyr}$ and $0.5 \mathrm{Gyr}$ respectively. Even considering the upper values for the age estimates, there are four stars estimated younger than $5 \mathrm{Gyr}$ is in both cases. Thus, the assessment of a sizeable fraction of young stars among the microlensed sample in the Galactic bulge appears robust.
\end{abstract}

Key words. stars: evolution - gravitational lensing: micro - Galaxy: bulge - methods: statistical - Galaxy: evolution

\section{Introduction}

Recently a debate on the age of the stars in the Galactic bulge has developed. Until few years ago, the picture of a Galactic bulge composed of old stars was commonly accepted. The evidence from Hubble Space Telescope photometry of the Galactic bulge main-sequence turnoff points toward an exclusively old stellar population, with ages greater than about $10 \mathrm{Gyr}$ (e.g. Zoccali et al. 2003; Brown et al. 2010; Clarkson et al. 2011; Valenti et al. 2013). The similarity between the Galactic bulge and Galactic globular clusters suggested an age estimate of 12-13 Gyr (Marín-Franch et al. 2009).

However, in recent years a different scenario coming from gravitational microlensing results was proposed. Bensby et al. $(2011,2013)$ obtained high-resolution spectra of 58 bulge mainsequence and subgiant stars, from which they derived measurements of $T_{\text {eff }}, \log g$, and [Fe/H]. Surprisingly, the comparison of the observations with theoretical isochrones revealed a significant fraction of young and intermediate-age stars. In fact, the Bensby et al. (2013) results showed the presence of 13 stars out

$\star$ Table 1 and Appendix A are available in electronic form at http: //www . aanda.org of $58(22 \%)$ younger than 5 Gyr and 31 stars (53\%) younger than 9 Gyr.

As a consequence of these results, there has been some effort in the literature to reconcile the two pictures. A first attempt was made by Nataf \& Gould (2012), who suggested that taking into account the possible Galactic bulge helium enhancement, the differences between the two sets of age estimates should vanish. However, this result was questioned by Bensby et al. (2013), who claimed that the effect of the helium enhancement is insufficient to contradict the conclusion of a substantial presence of stars at young and intermediate ages. Furthermore, a possible contamination of the microlensing sample by disk star and bias toward a lower age was proposed (Nataf 2013). However, none of these distortions seem to be large enough to refute the Bensby et al. (2013) findings. More recently, Ness et al. (2014) suggested that the discrepancy could be due to the different spatial distributions in the Galactic bulge of old and young stars.

Given the importance of the age estimates by Bensby et al. (2013) in the framework of Galactic bulge formation scenarios (see e.g. Ness et al. 2014, and references therein), we feel that an independent analysis of these results - adopting a different estimation technique and different theoretical stellar models and an analysis of potential systematic biases is mandatory. The 
purpose of the present work is to provide such an independent check adopting the SCEPtER pipeline (Valle et al. 2014, 2015b).

We also explore in detail the impact on these age estimates of changing some parameters or prescriptions required to compute a grid of stellar models that are not yet tightly constrained. In particular, we analysed the effect of a helium-rich Galactic bulge by computing a grid of stellar models with a helium-to-metal enrichment ratio $\Delta Y / \Delta Z$ much higher than the standard one (i.e. $\Delta Y / \Delta Z=5$ instead of 2). Moreover, we checked the systematic differences in age estimates due to the inclusion or not of the microscopic element diffusion in stellar evolution calculations, which is an important bias source in grid-based techniques (Valle et al. 2014, 2015b). Finally, we quantified the effect of changing the mixing-length calibration in stellar models.

The structure of the paper is the following. In Sect. 2 we discuss the estimation method and the stellar-model grids used in the process. The age estimates are presented in Sect. 3, and the analysis of the impact of changing the grid of models by taking into account a much higher helium abundance, by neglecting the element microscopic diffusion, and by increasing the mixinglength parameter is performed in Sect. 4. Some concluding remarks can be found in Sect. 5. Finally, a Monte Carlo validation of the adopted method for stars in evolutionary stages similar to those of the microlensed sample is presented in Appendix A.

\section{Methods}

The age estimates obtained in this paper are based on our recently developed pipeline SCEPtER, which is extensively described in Valle et al. $(2014,2015 b)$ and briefly summarized here. We let $\mathcal{S}$ be a star for which the following vector of observed quantities is available: $q^{\mathcal{S}} \equiv\left\{T_{\text {eff }, \mathcal{S}},[\mathrm{Fe} / \mathrm{H}]_{\mathcal{S}}, \log g_{\mathcal{S}}\right\}$. Then we let $\sigma=\left\{\sigma\left(T_{\text {eff }, \mathcal{S}}\right), \sigma\left([\mathrm{Fe} / \mathrm{H}]_{\mathcal{S}}\right), \sigma\left(\log g_{\mathcal{S}}\right)\right\}$ be the nominal uncertainty in the observed quantities. For each point $j$ on the estimation grid of stellar models, we define $q^{j} \equiv$ $\left\{T_{\text {eff }, j},[\mathrm{Fe} / \mathrm{H}]_{j}, \log g_{j}\right\}$. We let $\mathcal{L}_{j}$ be the likelihood function defined as

$\mathcal{L}_{j}=\left(\prod_{i=1}^{3} \frac{1}{\sqrt{2 \pi} \sigma_{i}}\right) \times \exp \left(-\frac{\chi^{2}}{2}\right)$,

where

$\chi^{2}=\sum_{i=1}^{3}\left(\frac{q_{i}^{\mathcal{S}}-q_{i}^{j}}{\sigma_{i}}\right)^{2}$.

The likelihood function is evaluated for each grid point within $3 \sigma$ of all the variables from $\mathcal{S}$; we let $\mathcal{L}_{\max }$ be the maximum value obtained in this step. The estimated stellar mass, radius, and age are obtained by averaging the corresponding quantity of all the models with likelihood greater than $0.95 \times \mathcal{L}_{\max }$.

The technique allows a Monte Carlo confidence interval to be constructed for the estimates. To this purpose a synthetic sample of $n$ stars is generated, following a multivariate normal distribution with vector of mean $q^{\mathcal{S}}$ and covariance matrix $\Sigma=\operatorname{diag}(\sigma)$. A value of $n=10000$ is usually adopted since it provides a fair balance between computation time and the accuracy of the results. The medians of the estimated mass and age for th $n$ objects are taken as the best estimate of the true values; the 16th and 84th quantiles of the $n$ values are adopted as a $1 \sigma$ confidence interval.

\subsection{Standard stellar models grid}

The standard estimation grid of stellar models was obtained using the FRANEC stellar evolution code (Degl'Innocenti et al. 2008; Tognelli et al. 2011), as it was adopted to compute the Pisa Stellar Evolution Data Base ${ }^{1}$ for low-mass stars (Dell'Omodarme et al. 2012; Dell'Omodarme \& Valle 2013).

The grid consists of stellar models in evolutionary stages from the zero-age main sequence (ZAMS) to the helium flash at the tip of the red giant branch (RGB). Models of age greater than 15 Gyr were excluded from the grid. Models were computed for stellar masses in the range $[0.60 ; 1.60] M_{\odot}$ with a step of $0.02 M_{\odot}$. The initial metallicity $[\mathrm{Fe} / \mathrm{H}]$ was assumed in the range $[-2.0 ; 0.55]$ with a step of 0.10 dex from -2.0 to -1.0 and 0.05 dex for $[\mathrm{Fe} / \mathrm{H}]>-1.0$. The solar scaled heavyelement mixture by Asplund et al. (2009) was adopted. For $[\mathrm{Fe} / \mathrm{H}]$ lower than -0.5 dex we accounted for an $\alpha$-elements enhancement $[\alpha / \mathrm{Fe}]=+0.3$. The initial helium abundance was obtained using the linear relation $Y=Y_{\mathrm{p}}+\frac{\Delta Y}{\Delta Z} Z$. The primordial ${ }^{4} \mathrm{He}$ abundance value $Y_{\mathrm{p}}=0.2485$ from WMAP was adopted (Cyburt et al. 2004; Steigman 2006; Peimbert et al. 2007a,b), assuming $\Delta Y / \Delta Z=2$ (Pagel \& Portinari 1998; Jimenez et al. 2003; Gennaro et al. 2010). The models were computed assuming our solar-scaled mixing-length parameter $\alpha_{\mathrm{ml}}=1.74$. Atomic diffusion was included adopting the coefficients given by Thoul et al. (1994) for gravitational settling and thermal diffusion. Convective core overshooting was not taken into account. Further details on the input of the code can be found in Valle et al. (2009, 2013a,b).

A Monte Carlo estimation of the expected errors and possible biases of our technique for stars similar to those in the microlensed bulge sample, adopting the available set of observational constraints, is reported in Appendix A. The analysis showed that the method is unbiased for the considered evolutionary phases.

The adopted estimation technique and the grid of stellar models differ under many aspects from those used by Bensby et al. (2013), therefore a confirmation of their finding would add considerable robustness to the age results. Bensby et al. $(2011,2013)$ adopted a maximum likelihood functional maximization, where the errors on the estimates are derived from the likelihood profiles. They used the stellar models by Yonsei-Yale isochrones (Demarque et al. 2004), which rely on input physics different from our own reference. In particular, they adopt the solar mixture from Grevesse et al. (1996), the low temperatures opacities by Alexander \& Ferguson (1994), the conductive opacities by Hubbard \& Lampe (1969), the OPAL equation of state in the 1996 version (Rogers et al. 1996), helium but no metal microscopic diffusion, and the cross sections listed in Bahcall (1989). Moreover, they take into account a mild convective core overshooting following the recipe described in Demarque et al. (2004).

\section{Age estimates}

The observational data - with their errors - of the 58 microlensed bulge stars studied in this paper have been obtained from Table 4 in Bensby et al. (2013). For each object, we estimated the mass and the age by means of the SCEPtER technique, as detailed in Sect. 2. Figure 1 and Table 1 show our results together with the observed $[\mathrm{Fe} / \mathrm{H}]$. Figure 2 shows the comparison between our age estimates and those by Bensby et al. (2013) (left panel), and

1 http://astro.df.unipi.it/stellar-models/ 


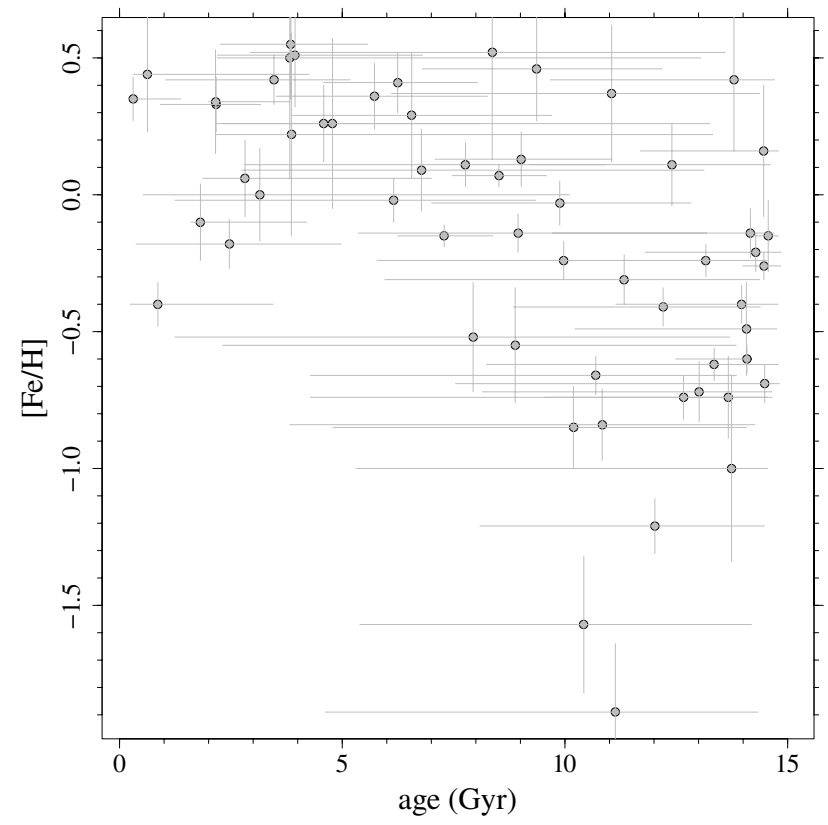

Fig. 1. $[\mathrm{Fe} / \mathrm{H}]$ versus SCEPtER age estimates for the 58 microlensed stars.

the age differences between the former and the latter as a function of the metallicity (right panel). The error bars on the age differences are obtained by adding in quadrature the errors provided by the two techniques. As one can see, the two independent results generally agree with each other, as it is apparent that all the differences are consistent with zero. A comparison of the two sets by means of a paired $t$-test showed a small mean difference of -0.08 Gyr (95\% confidence interval [ $-0.46 ; 0.30]$ Gyr), visible in Fig. 2.

This agreement is further confirmed by comparing the empirical cumulative distributions of stellar ages estimated by the two techniques, shown in Fig. 3. A two-sample KolmogorovSmirnov test did not reveal significant differences between the two empirical distributions $(D=0.105, p$-value $=0.92) \mathrm{im}$ plying that the age distribution can be considered consistent between the two estimation methods.

It is apparent from Fig. 3 that both the techniques independently estimate a sizeable fraction of young stars. We found 16 stars younger than 5 Gyr and 28 younger than 9 Gyr that is, $28 \%$ (95\% confidence interval $[17 \% ; 41 \%])$ and $48 \%$ (95\% confidence interval $[35 \% ; 62 \%]$ ) of the sample, respectively, whereas Bensby et al. (2013) found $13(22 \%)$ and $31(58 \%)$.

\section{Effect of changing the grid of stellar models}

The determination of stellar ages necessarily requires theoretical stellar models, which in turn depend on prescriptions and parameters that have not yet been tightly constrained (Valle et al. 2013a,b). As a consequence, the resulting age estimates are affected by non-negligible uncertainties and, possibly, biases. In recent years, several studies have been devoted to the analysis of the performance of grid based techniques (e.g. Gai et al. 2011; Basu et al. 2012; Mathur et al. 2012). In this perspective, in Valle et al. $(2014,2015 b)$ we carried out a detailed statistical analysis of the main biases affecting any grid-based recovery procedure. In this section we will discuss the impact on the age estimate of the 58 microlensed bulge stars of increasing the initial helium abundance, switching off microscopic diffusion, and changing the mixing-length calibration adopted to compute the stellar models. The main aim of the test is to check the robustness of the assessment of a sizeable fraction of young bulge stars.

\subsection{Effect of increasing the initial helium abundance}

Nataf \& Gould (2012) suggested that the discrepancy in age between Bensby et al. (2013) results and those inferred by photometric data would vanish whenever an enhancement in the helium content of the bulge stars was addressed. Nataf \& Gould (2012), studying the effect on theoretical isochrones of helium abundance variations, discussed the systematic effects on age estimates that could arise when observational data were analysed with standard instead of helium-enhanced isochrones. In particular, they found that the predicted trend of the difference between the true absolute magnitude and the spectroscopically inferred one should be positively correlated with the spectroscopically inferred stellar mass, a trend that they found also present in the Bensby et al. (2011) data. Thus they claimed that a value of helium-to-metal enrichment ratio of $\Delta Y / \Delta Z \approx 5$ would reconcile the two sets of age estimates. In contrast, Bensby et al. (2013) showed that the discussed trend is no longer present in the extended stellar sample; they rejected the hypothesis by Nataf \& Gould (2012) and concluded that a sizeable sample of young and intermediate age stars would still be present even assuming high helium abundances. However, neither Nataf \& Gould (2012) nor Bensby et al. (2013) performed a statistically detailed comparison of the age estimates obtained with different values of the original helium abundance. Moreover, the impact of assuming a different initial helium value is strongly dependent on the observational quantities used to constrain the ages. As an example, in Valle et al. (2015b) we have recently shown that in the presence of asteroseismic constraints the impact on age estimates of a change by \pm 1 in $\Delta Y / \Delta Z$ is indeed negligible, while the contrary occurs if mass and radius are available (Valle et al. 2015a).

Because of its potential impact in reducing the number of estimated young bulge stars, the effect of a large increase in the initial helium content is worthy of a detailed quantitative analysis. Therefore, we computed a non-standard grid of models with the same parameters or input physics and the same values of the metallicity $Z$ as in the standard grid, but by significantly increasing the helium-to-metal enrichment ratio, i.e. $\Delta Y / \Delta Z=5$ instead of 2 . Then we repeated the age estimate by adopting this helium-enhanced grid in the SCEPtER pipeline. To account for possible correlations among the estimates given by the standard and helium-enhanced grids, some care was needed in the comparison. In fact, the naive differences of the two estimates (and their errors) could be obtained by subtracting the estimates provided by the standard and helium-enhanced grids and by adding in quadrature the errors in the single estimates. However, since such an approach largely overestimates the final errors, we preferred to follow a more accurate procedure. For each star, we obtained $N=10000$ artificially perturbed stars adding a Gaussian noise to the observables as detailed in Sect. 2. The artificial star ages are then estimated by means of the SCEPtER pipeline with both grids. For each of the $N$ artificial stars, we evaluated the difference between the age returned by the helium-enhanced and the standard grids. The median of these differences was adopted as best estimate and the 16th and 84th quantiles were adopted as $1 \sigma$ error.

The results of this analysis are given in Fig. 4. The left panel of the figure shows the differences in the estimates computed 

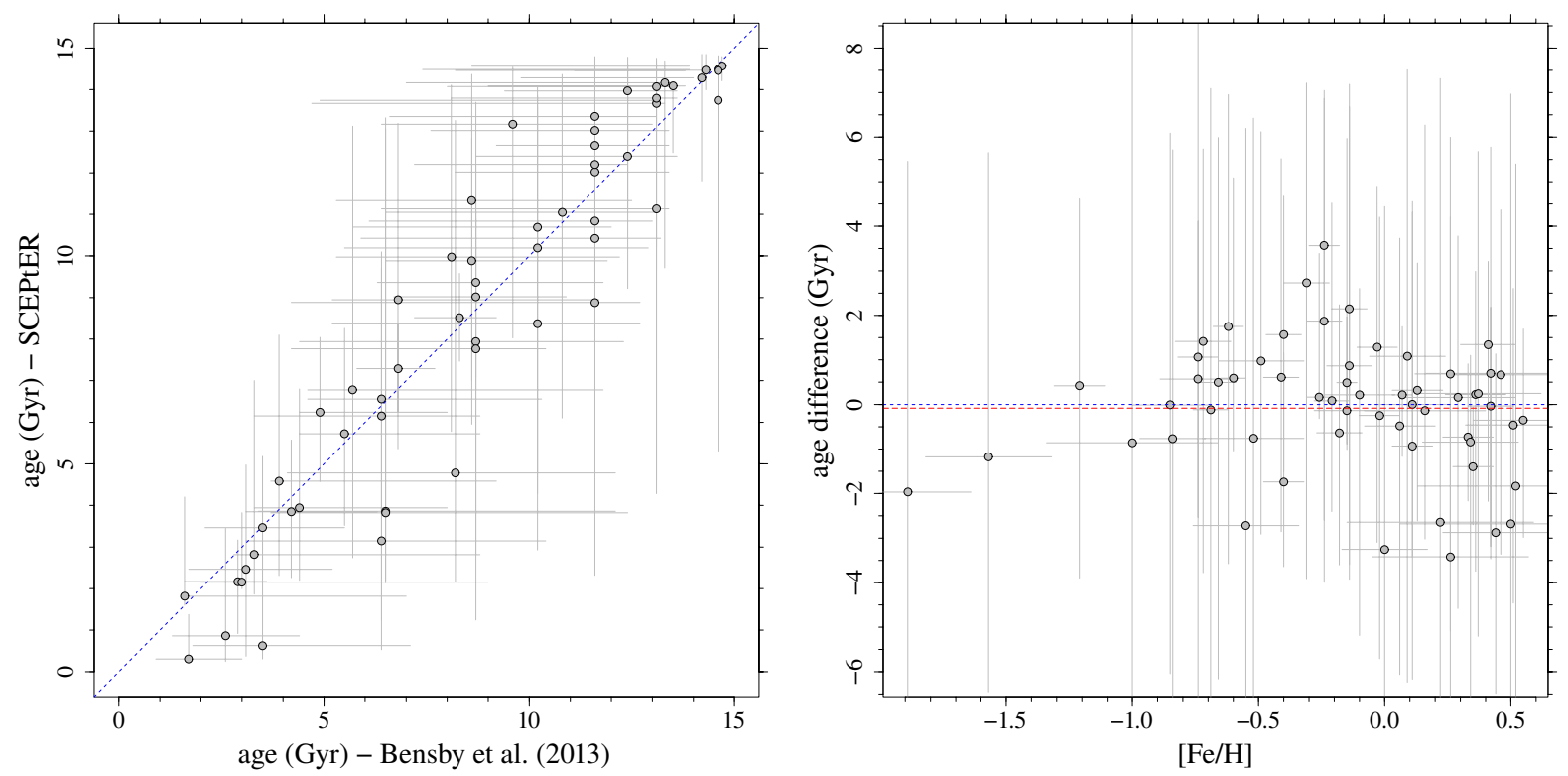

Fig. 2. Left: SCEPtER age estimates for the 58 microlensed stars versus the Bensby et al. (2013) estimates. Right: difference in age estimates between the two techniques versus $[\mathrm{Fe} / \mathrm{H}]$. A positive value means that the SCEPtER age is greater than the corresponding age estimated by Bensby et al. (2013). The red dashed line marks the mean difference of -0.08 Gyr between the two sets of age estimates.

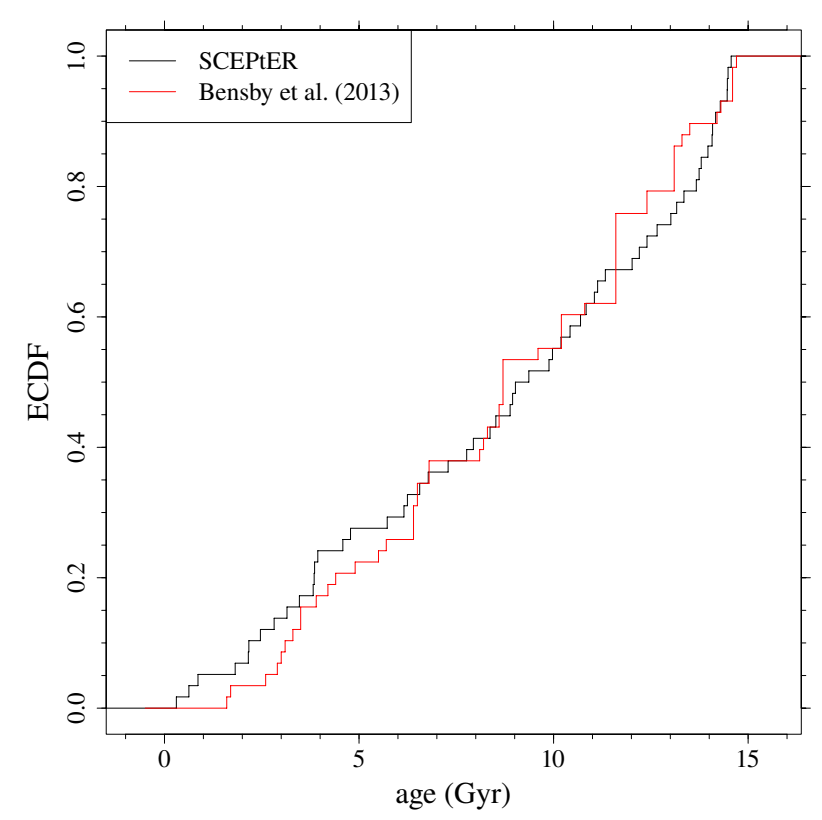

Fig. 3. Empirical cumulative distribution functions for the 58 estimated ages obtained by SCEPtER (black line) and by Bensby et al. (2013) (red line).

with the helium-enhanced and the standard grid of models. It appears that the change in the initial helium content has a little influence in age estimates. Adopting the helium-enhanced grid, the mean increase in age estimates is $0.35 \mathrm{Gyr}(95 \%$ confidence interval $[0.26 ; 0.44] \mathrm{Gyr})$. As expected, the influence of the initial helium abundance change is greater at high metallicity. The effect is shown in the figure by superimposing a LOESS smoother ${ }^{2}$

\footnotetext{
2 A LOESS (LOcal regrESSion) smoother is a non-parametric locally weighted polynomial regression technique that is often used to highlight the underlying trend of scattered data (see e.g. Feigelson \& Babu 2012; Venables \& Ripley 2002).
}

on the data: the higher the metallicity, the larger the discrepancy, until a plateau of about $0.6 \mathrm{Gyr}$ for $[\mathrm{Fe} / \mathrm{H}]>0$. However, such an increase in the age estimates is too small to rule out the evidence of a young component in the microlensed population.

As one can see in Fig. 2, the error bars on the age estimates are relevant. An important point to address is thus whether the previous finding might result simply from a fluctuation. Since we are mainly interested in checking the robustness of the identification of a sizeable fraction of young stars, we further analysed our results by considering as age estimates those provided by the upper values of the error bars rather than the mean values. With such a choice, the probability of obtaining by chance an age older than the adopted one is $16 \%$. The right panel in Fig. 4 shows the empirical cumulative density function for the upper values obtained by SCEPtER assuming standard and heliumenhanced grids of models, and by Bensby et al. (2013). The three cumulative distributions closely agree up to 12 Gyr. All three sets of estimates contain 15 stars younger than 9 Gyr $(26 \%$ of the sample, $95 \%$ confidence interval $[16 \% ; 39 \%])$. For stars younger than $5 \mathrm{Gyr}$, the estimates assuming standard helium abundance show the presence of seven such objects (12\% of the sample, 95\% confidence interval [5\%; 24\%]), while there are four (7\% of the sample, $95 \%$ confidence interval $[2 \% ; 18 \%])$ in the helium-enhanced computations.

Thus, even in the most conservative scenario in which we contemporaneously adopt a high initial helium abundance and the upper values of the age estimates, there is strong evidence of a young population among the microlensed bulge stars.

\subsection{Effect of neglecting microscopic diffusion}

An important source of systematic bias in the age estimate of dwarf stars is the treatment of element microscopic diffusion adopted in stellar model computations. Depending on its efficiency, this process affects the evolution of both the surface chemical abundance - and thus $[\mathrm{Fe} / \mathrm{H}]$ - and the internal structure of low-mass stars. As a direct consequence, the stellar parameters determined by comparing theoretical models and 

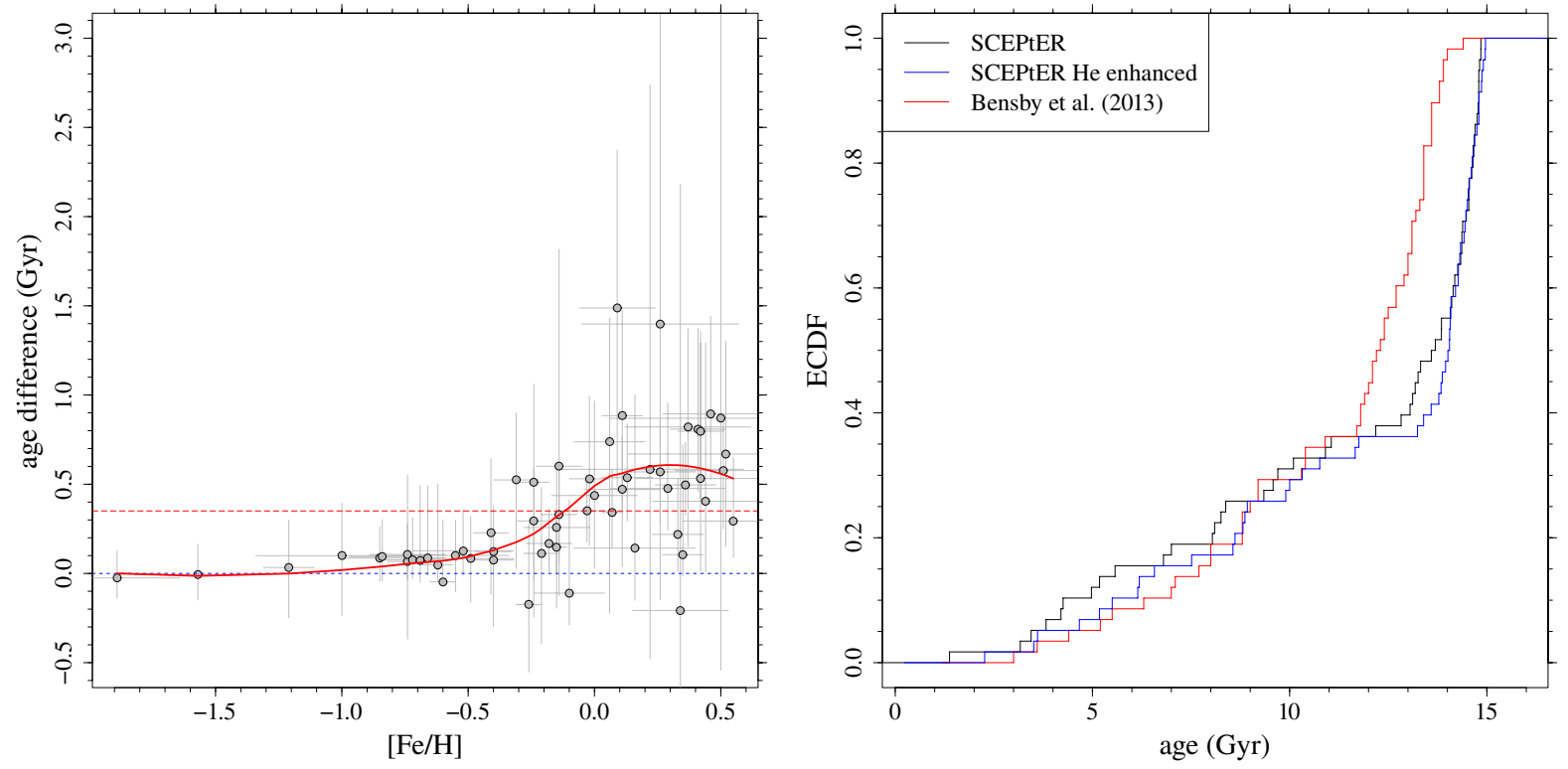

Fig. 4. Left: differences between the age estimates obtained by adopting the grid with $\Delta Y / \Delta Z=5$ (helium-enhanced) and that with $\Delta Y / \Delta Z=2$ (standard). The red dashed line marks the mean of the differences, while the solid red line is a LOESS smoother of the data (see text). Right: empirical cumulative distribution functions for the upper limits of the estimated ages obtained by SCEPtER assuming the standard initial helium value (black line), the helium-enhanced scenario (blue line), and for the ages estimated by Bensby et al. (2013) (red line).

observations depend on the diffusion efficiency adopted. In Valle et al. $(2014,2015 b)$ we showed that grid-based estimates of mass, radius, and age are significantly different between grids of models that take into account diffusion and those that neglect it. From the observational point of view, microscopic diffusion has been proved to be efficient in the Sun (see e.g. Bahcall et al. 2001), while its actual efficiency in Galactic globular cluster stars is debated (see e.g. Gruyters et al. 2014; Nordlander et al. 2012; Gratton et al. 2011; Korn et al. 2007).

The Bensby et al. (2013) results are based on stellar models that consider only the diffusion of helium and not that of metals. On the contrary, our standard grid follows the diffusion of helium and metals, as mentioned in Sect. 2.1. Since our main aim is to verify whether the identification of young stars is robust against theoretical biases, we think that it is worth exploring the effects of completely neglecting microscopic diffusion in stellar calculations. Such a choice would in fact maximize age estimates, the other parameters being fixed, and consequently minimize the sample of young stars. We computed a non-standard grid of models with exactly the same characteristics as the standard model but without the inclusion of a diffusion mechanism. Then, we repeated the age estimate by adopting this non-standard grid in the SCEPtER pipeline. The differences between the age estimates with and without diffusion have been computed in the same way as discussed in Sect. 4.1 for the helium-rich case.

The results are shown in the left panel of Fig. 5. As expected (see e.g. Valle et al. 2015b), the neglect of diffusion in stellar models leads to older ages. However, such an effect gets smaller and smaller at decreasing ages owing to the long time scale of diffusion processes. The maximum increase of the estimated age is about $2.8 \mathrm{Gyr}$ at $8 \mathrm{Gyr}$ and lower than $1.5 \mathrm{Gyr}$ for stars younger than 6 Gyr. The mean age increase is indeed much smaller being of $0.40 \mathrm{Gyr}$ (95\% confidence interval $[0.17 ; 0.63]$ Gyr) for stars younger than 5 Gyr. The coloured region in the figure highlights stars with estimated ages higher than 10 Gyr. The decreasing trend in the age differences for ages higher than about $10 \mathrm{Gyr}$ is an edge-effect, a common feature of any grid-based technique, as extensively discussed in Valle et al. (2014, 2015b). Such an effect is the consequence of the lack of models older than 15 Gyr in the grid, which thus limits the possibility of age overestimation for these old stars.

The effect of the diffusion on age estimates can also be seen in the right panel of Fig. 5, which shows the empirical cumulative density functions for the upper values for the age estimates obtained considering or neglecting microscopic diffusion.

It is apparent that even neglecting the microscopic diffusion into stellar model computations, there is no evidence against the conclusion of the presence of a relevant young stellar population. Even in the most unfavourable case in which the non-standard grid without diffusion and the upper values of the age estimates are used there are 4 (7\% of the sample, $95 \%$ confidence interval [2\%; 18\%]) younger than 5 Gyr and 11 (19\% of the sample, $95 \%$ confidence interval $[10 \% ; 32 \%])$ younger than 9 Gyr.

\subsection{Effect of increasing the superadiabatic convection efficiency}

Another source of systematic bias in age determination of dwarf stars is the efficiency of convective transport in superadiabatic regimes adopted in stellar computations. As is well known, modern evolutionary codes still have to rely on oversimplified treatments of superadiabatic convection which depend on free parameters to be calibrated with observations. The most commonly adopted treatment is the mixing-length theory (Böhm-Vitense 1958), where the efficiency of convective transport depends on the free parameter $\alpha_{\mathrm{ml}}$, usually calibrated with the Sun. A solar calibrated mixing-length has been adopted both in our standard models and in those used by Bensby et al. (2013). However, there is no a priori compelling reason to guarantee that a solar calibration also holds in evolutionary phases and/or masses different from that of the Sun. Moreover, there are indications that a slightly higher value $\left(\alpha_{\mathrm{ml}}=1.90\right)$ than the solar calibrated is in better agreement with the colour-magnitude diagrams of old and intermediate age Galactic globular clusters (see e.g. 

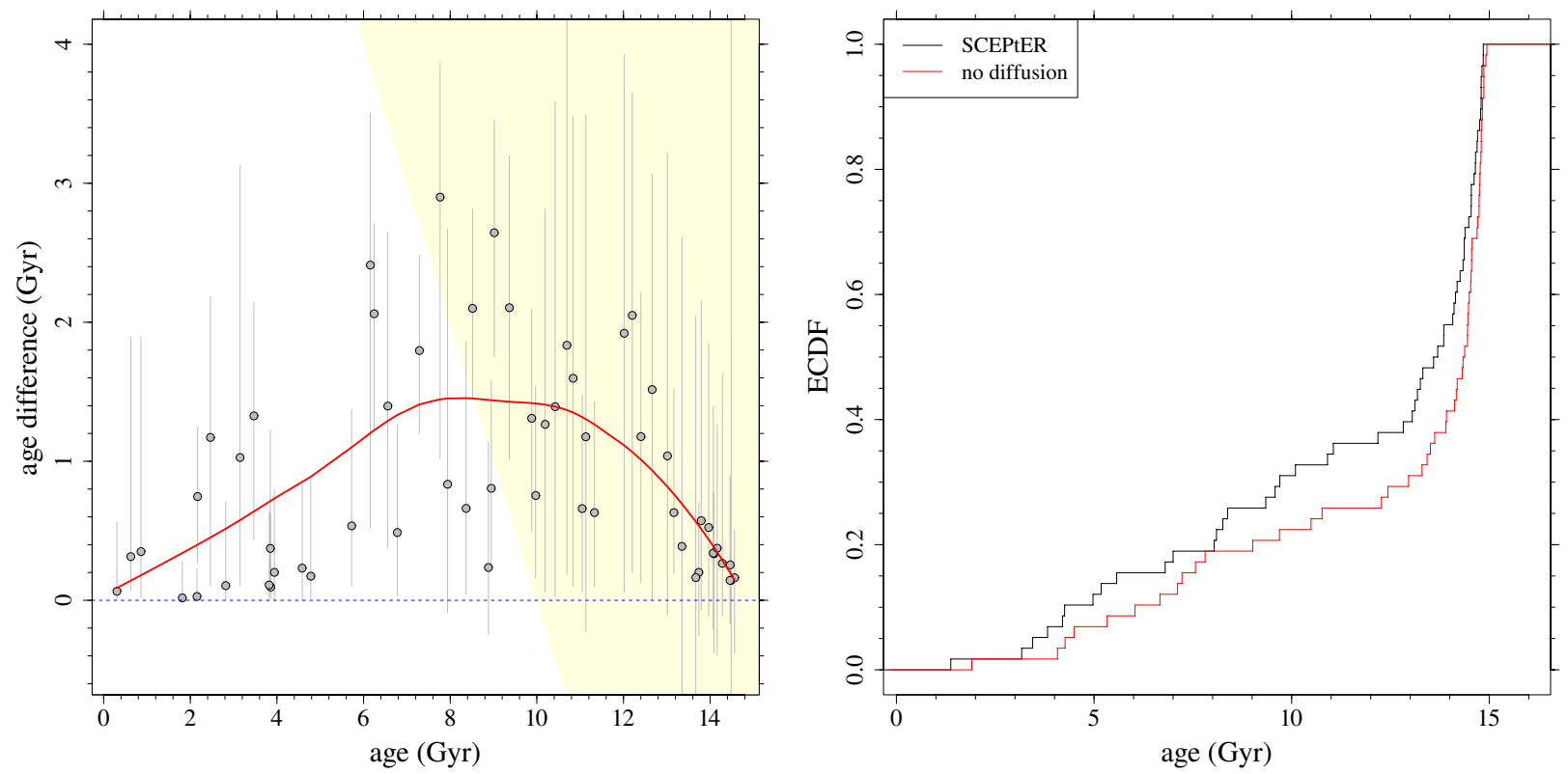

Fig. 5. Left: differences in age estimates assuming the grid that neglects microscopic diffusion and the standard one. The solid red line is a LOESS smoother of the data (see text). The yellow region shows the stars older than $10 \mathrm{Gyr}$. Right: empirical cumulative distribution functions for the upper limits of the estimated ages obtained by SCEPtER adopting the grid with element diffusion (black line), and the grid that neglects it (red line).
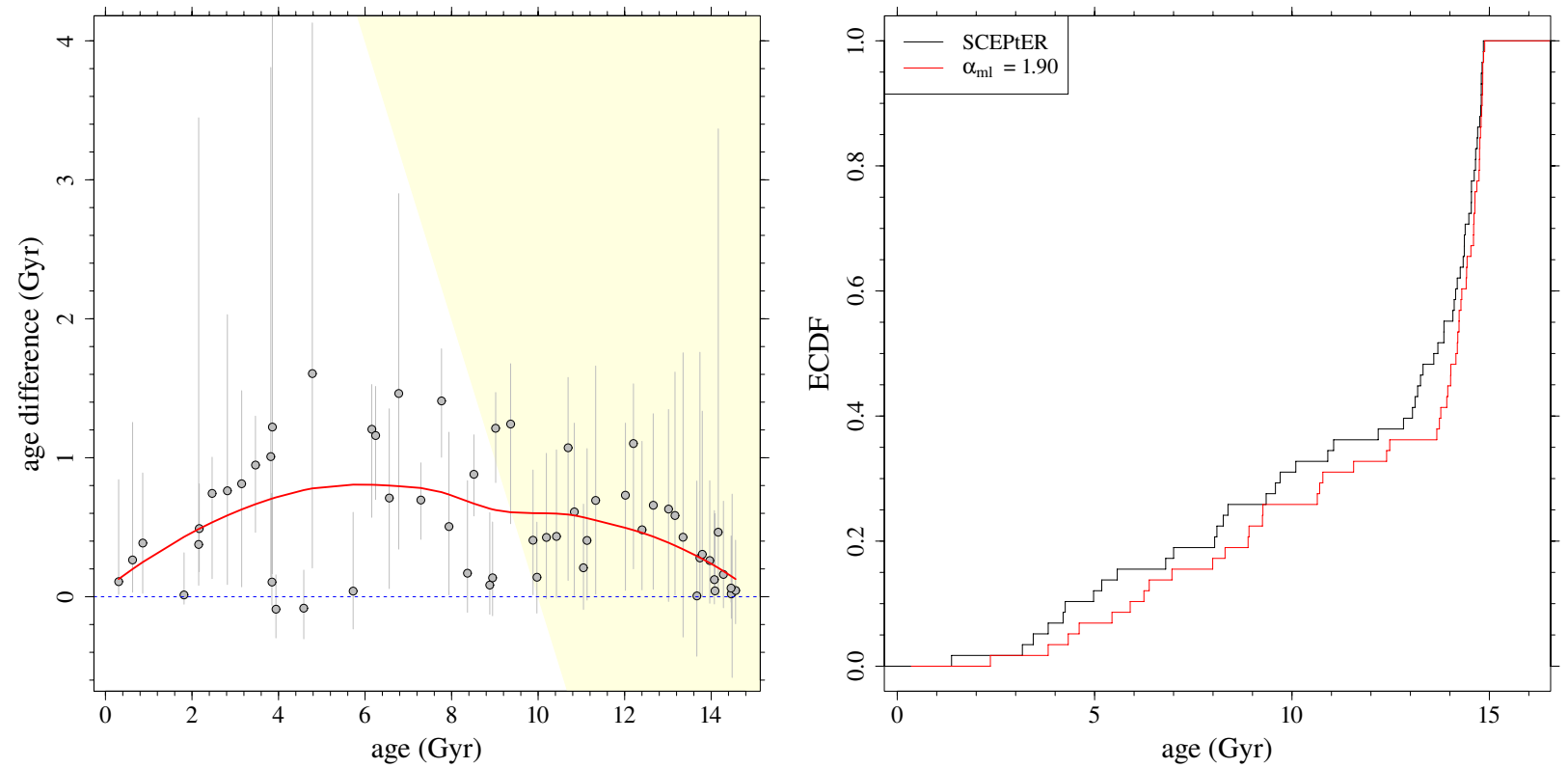

Fig. 6. Left: same as in the left panel of Fig. 5, but for age differences due to assuming the grid with $\alpha_{\mathrm{ml}}=1.90$ and the reference case with $\alpha_{\mathrm{ml}}=1.74$. Right: empirical cumulative distribution functions for the upper limits of the estimated ages obtained by SCEPtER adopting the standard grid with $\alpha_{\mathrm{ml}}=1.74$ (black line), and that with $\alpha_{\mathrm{ml}}=1.90$ (red line).

Dell'Omodarme et al. 2012). For this reason we think that it is worth discussing the effect on the age estimates of a change in the mixing-length value. As in the two previous tests, we focused only on a variation that would increase stellar ages, thus reducing the number of young bulge stars.

We computed a non-standard grid of models with the same characteristics of the standard one but computed with $\alpha_{\mathrm{ml}}=$ 1.90. Then, we repeated the age estimate by adopting this nonstandard grid in the SCEPtER pipeline. The differences in ages between the $\alpha_{\mathrm{ml}}=1.90$ and the $\alpha_{\mathrm{ml}}=1.74$ (solar calibrated) cases have been computed as in the previous tests (see Sects. 4.1 and 4.2). The results are presented in Fig. 6. The age increase due to the higher mixing-length value is lower than the increase due to neglecting microscopic diffusion, and its maximum is about 1.5 Gyr. For stars younger than 5 Gyr, the mean increase in age estimate is $0.54 \mathrm{Gyr}$ (95\% confidence interval [0.28; $0.81]$ Gyr).

The right panel of Fig. 6 shows the empirical cumulative density functions for the upper values of the age estimates obtained with grids of stellar models with the two considered 
values of $\alpha_{\mathrm{ml}}$. The differences between the two distributions are small. Even in the most unfavourable case, i.e. simultaneously adopting the grid with $\alpha_{\mathrm{ml}}=1.90$ and the upper values of the age, estimates provide 4 ( $7 \%$ of the sample, $95 \%$ confidence interval $[2 \% ; 18 \%])$ younger than $5 \mathrm{Gyr}$ and 13 (22\% of the sample, $95 \%$ confidence interval $[13 \% ; 36 \%]$ ) younger than 9 Gyr.

In conclusion, even accounting for a higher mixing-length value, the presence of a relevant young stellar population is confirmed.

\section{Conclusions}

We independently verified the results by Bensby et al. (2013) on the age of 58 microlensed dwarf and subgiant stars in the Galactic bulge by means of completely different stellar models and estimation techniques.

To this purpose we used the SCEPtER pipeline (Valle et al. 2014, 2015b), which allows mass, radius, and age of single stars to be estimated by relying on a fine grid of precomputed stellar tracks and a set of observational constraints. Here we adopted the effective temperature, the surface gravity, and $[\mathrm{Fe} / \mathrm{H}]$ provided by Bensby et al. (2013). The standard grid was composed by models from ZAMS up to the helium flash at the RGB tip, for mass in the range $[0.60 ; 1.60] M_{\odot}$.

The performance of the estimation technique for the considered evolutionary phases was assessed by means of a Monte Carlo simulation. The results of this test showed that the adopted method is unbiased and it can be safely employed for the required age estimations.

We confirmed the results by Bensby et al. (2013), computed adopting a different estimation technique and a different grid of stellar models. This confirmation adds considerable robustness to the age estimates and in particular to the discovery of a sizeable fraction of young bulge stars. We found in fact that the analysed sample of microlensed stars contains 16 stars (about one third of the sample) younger than $5 \mathrm{Gyr}$ and 27 (about one half of the sample) younger than 9 Gyr. Even conservatively accounting for the errors in the obtained stellar ages, assuming the upper values as best age estimates, we found that 15 stars were definitely younger than 9 Gyr.

Given the importance of the result and its widespread implications, we analysed in detail possible biases in the age estimates due to some assumptions adopted in computing the grid of stellar models. We first checked if helium rich models would be able to rule out the evidence of the presence of young and intermediate age stars as suggested by Nataf \& Gould (2012). To this purpose we repeated the age estimation procedure relying on a helium-enhanced grid of models computed on purpose by adopting a helium-to-metal enrichment ratio $\Delta Y / \Delta Z=5$. The result was a median increase in the age of the stars with $[\mathrm{Fe} / \mathrm{H}]>0$ by about $0.6 \mathrm{Gyr}$, largely insufficient to reconcile the stellar age estimates with the picture of a totally old bulge suggested by photometric studies of the main-sequence turnoff (e.g. Zoccali et al. 2003; Brown et al. 2010; Clarkson et al. 2011; Valenti et al. 2013). To exclude the possibility that the results could arise only by a fluctuation, we also verified the robustness of our finding assuming the $1 \sigma$ upper values of age estimates as best values rather then the mean ones. Even with this extreme choice and adopting the helium-enhanced grid of stellar models there is evidence of 4 and 15 stars younger than 5 Gyr and 9 Gyr, respectively.

Another important source of systematic bias in the age estimate of dwarf stars is the treatment of element diffusion (Valle et al. 2014, 2015b). We verified that even neglecting the diffusion in the stellar models adopted in the estimate procedure is not able to increase the age enough to rule out the presence of a young and intermediate age population. We obtained a maximum increase of about 1 Gyr for stars younger than 5 Gyr and of about 2.8 Gyr for stars with ages of about 8 Gyr. The mean age increase is indeed much smaller, about 0.40 Gyr for stars younger than 5 Gyr. Even in the most unfavourable case in which the upper values of the age estimates are used, the number of stars younger than 5 and 9 Gyr are, respectively, 4 and 11 .

Finally, we checked the effect of changing the calibration of the mixing-length from $\alpha_{\mathrm{ml}}=1.74$ (our solar value) to $\alpha_{\mathrm{ml}}=1.90-$ a value calibrated on the RGB colour of old and intermediate age clusters - in the stellar models used to estimate the age. As in the previous cases, the resulting age increase is not old enough to reject in a statistically meaningful way the presence of young bulge stars. In fact, we found a maximum age increase of about $1.5 \mathrm{Gyr}$, and a mean increase of $0.54 \mathrm{Gyr}$ for stars younger than 5 Gyr. Even adopting the upper values of the age estimates, the stars younger than 5 and 9 Gyr are, respectively, 4 and 13.

In conclusion, the presence of a young and intermediate age population among the microlensed bulge appears very robust and statistically confirmed.

Acknowledgements. We thank the anonymous referee for the valuable comments. This work has been supported by PRIN-MIUR 2010-2011 (Chemical and dynamical evolution of the Milky Way and Local Group galaxies, PI F. Matteucci) and PRIN-INAF 2012 (The M4 Core Project with Hubble Space Telescope, PI L. Bedin).

\section{References}

Alexander, D. R., \& Ferguson, J. W. 1994, ApJ, 437, 879

Asplund, M., Grevesse, N., Sauval, A. J., \& Scott, P. 2009, ARA\&A, 47, 481

Bahcall, J. N. 1989, Neutrino astrophysics (Cambridge University Press)

Bahcall, J. N., Pinsonneault, M. H., \& Basu, S. 2001, ApJ, 555, 990

Basu, S., Verner, G. A., Chaplin, W. J., \& Elsworth, Y. 2012, ApJ, 746, 76

Bensby, T., Adén, D., Meléndez, J., et al. 2011, A\&A, 533, A134

Bensby, T., Yee, J. C., Feltzing, S., et al. 2013, A\&A, 549, A147

Böhm-Vitense, E. 1958, Z. Astrophys., 46, 108

Brown, T. M., Sahu, K., Anderson, J., et al. 2010, ApJ, 725, L19

Casagrande, L., Schönrich, R., Asplund, M., et al. 2011, A\&A, 530, A138

Clarkson, W. I., Sahu, K. C., Anderson, J., et al. 2011, ApJ, 735, 37

Cyburt, R. H., Fields, B. D., \& Olive, K. A. 2004, Phys. Rev. D, 69, 123519

Degl'Innocenti, S., Prada Moroni, P. G., Marconi, M., \& Ruoppo, A. 2008, Ap\&SS, 316, 25

Dell'Omodarme, M., \& Valle, G. 2013, The R Journal, 5, 108

Dell'Omodarme, M., Valle, G., Degl'Innocenti, S., \& Prada Moroni, P. G. 2012 A\&A, 540, A26

Demarque, P., Woo, J.-H., Kim, Y.-C., \& Yi, S. K. 2004, ApJS, 155, 667

Feigelson, E. D., \& Babu, G. J. 2012, Modern Statistical Methods for Astronomy with $\mathrm{R}$ applications (Cambridge University Press)

Gai, N., Basu, S., Chaplin, W. J., \& Elsworth, Y. 2011, ApJ, 730, 63

Gennaro, M., Prada Moroni, P. G., \& Degl'Innocenti, S. 2010, A\&A, 518, A13

Gratton, R. G., Johnson, C. I., Lucatello, S., D’Orazi, V., \& Pilachowski, C. 2011, A\&A, 534, A72

Grevesse, N., Noels, A., \& Sauval, A. J. 1996, in Cosmic Abundances, eds. S. S. Holt, \& G. Sonneborn, ASP Conf. Ser., 99, 117

Gruyters, P., Nordlander, T., \& Korn, A. J. 2014, A\&A, 567, A72

Hubbard, W. B., \& Lampe, M. 1969, ApJS, 18, 297

Jimenez, R., Flynn, C., MacDonald, J., \& Gibson, B. K. 2003, Science, 299, 1552

Jørgensen, B. R., \& Lindegren, L. 2005, A\&A, 436, 127

Korn, A. J., Grundahl, F., Richard, O., et al. 2007, ApJ, 671, 402

Marín-Franch, A., Aparicio, A., Piotto, G., et al. 2009, ApJ, 694, 1498

Mathur, S., Metcalfe, T. S., Woitaszek, M., et al. 2012, ApJ, 749, 152

Nataf, D. M. 2013, ArXiv e-prints [arXiv: 1307.3612]

Nataf, D. M., \& Gould, A. P. 2012, ApJ, 751, L39

Ness, M., Debattista, V. P., Bensby, T., et al. 2014, ApJ, 787, L19

Nordlander, T., Korn, A. J., Richard, O., \& Lind, K. 2012, ApJ, 753, 48 
Pagel, B. E. J., \& Portinari, L. 1998, MNRAS, 298, 747

Peimbert, M., Luridiana, V., \& Peimbert, A. 2007a, ApJ, 666, 636

Peimbert, M., Luridiana, V., Peimbert, A., \& Carigi, L. 2007b, in From Stars

to Galaxies: Building the Pieces to Build Up the Universe, eds. A. Vallenari,

R. Tantalo, L. Portinari, \& A. Moretti, ASP Conf. Ser., 374, 81

Rogers, F. J., Swenson, F. J., \& Iglesias, C. A. 1996, ApJ, 456, 902

Steigman, G. 2006, Int. J. Mod. Phys. E, 15, 1

Thoul, A. A., Bahcall, J. N., \& Loeb, A. 1994, ApJ, 421, 828

Tognelli, E., Prada Moroni, P. G., \& Degl'Innocenti, S. 2011, A\&A, 533, A109

Valenti, E., Zoccali, M., Renzini, A., et al. 2013, A\&A, 559, A98

Valle, G., Marconi, M., Degl'Innocenti, S., \& Prada Moroni, P. G. 2009, A\&A, 507,1541
Valle, G., Dell'Omodarme, M., Prada Moroni, P. G., \& Degl'Innocenti, S. 2013a, A\&A, 549, A50

Valle, G., Dell'Omodarme, M., Prada Moroni, P. G., \& Degl'Innocenti, S. 2013b, A\&A, 554, A68

Valle, G., Dell'Omodarme, M., Prada Moroni, P. G., \& Degl'Innocenti, S. 2014, A\&A, 561, A125

Valle, G., Dell'Omodarme, M., Prada Moroni, P. G., \& Degl'Innocenti, S. 2015a, A\&A, submitted

Valle, G., Dell'Omodarme, M., Prada Moroni, P. G., \& Degl'Innocenti, S. 2015b, A\&A, 575, A12

Venables, W., \& Ripley, B. 2002, Modern applied statistics with S, Statistics and computing (Springer)

Zoccali, M., Renzini, A., Ortolani, S., et al. 2003, A\&A, 399, 931

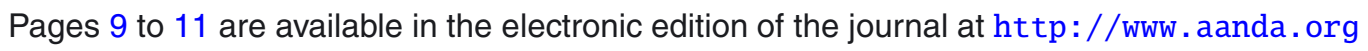


G. Valle et al.: On the age of Galactic bulge microlensed dwarf and subgiant stars

Table 1. Ages and masses determined by the SCEPtER pipeline.

\begin{tabular}{|c|c|c|c|c|c|c|c|c|}
\hline Object & $\begin{array}{l}\text { Age } \\
\text { (Gyr) }\end{array}$ & $\begin{array}{c}-1 \sigma \\
(\mathrm{Gyr})\end{array}$ & $\begin{array}{c}1 \sigma \\
(\mathrm{Gyr})\end{array}$ & $\begin{array}{l}\text { Mass } \\
\left(M_{\odot}\right)\end{array}$ & $\begin{array}{l}-1 \sigma \\
\left(M_{\odot}\right)\end{array}$ & $\begin{array}{c}1 \sigma \\
\left(M_{\odot}\right)\end{array}$ & {$[\mathrm{Fe} / \mathrm{H}]$} & $\Delta[\mathrm{Fe} / \mathrm{H}]$ \\
\hline OGLE-2012-BLG-1156 & 11.1 & 6.5 & 3.2 & 0.76 & 0.05 & 0.09 & -1.89 & 0.25 \\
\hline OGLE-2011-BLG-0969 & 10.4 & 5.0 & 3.8 & 0.79 & 0.05 & 0.13 & -1.57 & 0.25 \\
\hline MOA-2010-BLG-285 & 12.0 & 3.9 & 2.5 & 0.79 & 0.04 & 0.07 & -1.21 & 0.10 \\
\hline MOA-2010-BLG-078 & 13.7 & 8.4 & 0.8 & 0.82 & 0.04 & 0.25 & -1.00 & 0.34 \\
\hline MOA-2011-BLG-104 & 10.2 & 5.4 & 3.9 & 0.83 & 0.05 & 0.13 & -0.85 & 0.15 \\
\hline OGLE-2012-BLG-0270 & 10.8 & 7.0 & 3.4 & 0.81 & 0.04 & 0.07 & -0.84 & 0.13 \\
\hline MOA-2012-BLG-187 & 12.7 & 3.1 & 1.9 & 0.81 & 0.03 & 0.05 & -0.74 & 0.08 \\
\hline MOA-2009-BLG-493 & 13.7 & 9.4 & 1.0 & 0.72 & 0.04 & 0.08 & -0.74 & 0.15 \\
\hline OGLE-2009-BLG-076 & 13.0 & 4.9 & 1.6 & 0.80 & 0.04 & 0.04 & -0.72 & 0.11 \\
\hline MOA-2009-BLG-133 & 14.5 & 6.9 & 0.3 & 0.74 & 0.02 & 0.06 & -0.69 & 0.07 \\
\hline OGLE-2012-BLG-0563 & 10.7 & 6.4 & 3.2 & 0.81 & 0.03 & 0.04 & -0.66 & 0.07 \\
\hline OGLE-2012-BLG-1279 & 13.4 & 5.1 & 1.4 & 0.78 & 0.02 & 0.03 & -0.62 & 0.06 \\
\hline MOA-2010-BLG-167 & 14.1 & 1.6 & 0.1 & 0.82 & 0.00 & 0.03 & -0.60 & 0.05 \\
\hline MOA-2012-BLG-532 & 8.9 & 6.6 & 5.0 & 0.90 & 0.10 & 0.45 & -0.55 & 0.21 \\
\hline MOA-2009-BLG-475 & 7.9 & 6.7 & 5.8 & 0.84 & 0.06 & 0.08 & -0.52 & 0.20 \\
\hline MACH-1999-BLG-022 & 14.1 & 3.9 & 0.7 & 0.83 & 0.04 & 0.08 & -0.49 & 0.17 \\
\hline OGLE-2012-BLG-1217 & 12.2 & 3.4 & 2.2 & 0.83 & 0.03 & 0.04 & -0.41 & 0.07 \\
\hline MOA-2010-BLG-049 & 14.0 & 2.8 & 0.8 & 0.84 & 0.02 & 0.06 & -0.40 & 0.07 \\
\hline MOA-2010-BLG-446 & 0.9 & 0.6 & 2.6 & 1.01 & 0.05 & 0.05 & -0.40 & 0.08 \\
\hline OGLE-2008-BLG-209 & 11.3 & 5.4 & 3.0 & 0.90 & 0.06 & 0.18 & -0.31 & 0.09 \\
\hline MOA-2011-BLG-090 & 14.5 & 0.5 & 0.4 & 0.86 & 0.02 & 0.00 & -0.26 & 0.05 \\
\hline OGLE-2012-BLG-1526 & 13.2 & 5.1 & 1.4 & 0.88 & 0.02 & 0.13 & -0.24 & 0.06 \\
\hline MOA-2012-BLG-391 & 10.0 & 4.2 & 4.1 & 0.95 & 0.09 & 0.15 & -0.24 & 0.07 \\
\hline MOA-2009-BLG-489 & 14.3 & 2.5 & 0.6 & 0.86 & 0.02 & 0.05 & -0.21 & 0.07 \\
\hline MOA-2011-BLG-174 & 2.5 & 2.1 & 2.5 & 1.05 & 0.05 & 0.07 & -0.18 & 0.09 \\
\hline OGLE-2012-BLG-1534 & 7.3 & 1.0 & 1.1 & 1.04 & 0.05 & 0.05 & -0.15 & 0.04 \\
\hline MOA-2012-BLG-202 & 14.6 & 0.4 & 0.2 & 0.88 & 0.02 & 0.02 & -0.15 & 0.13 \\
\hline OGLE-2012-BLG-0617 & 14.2 & 4.5 & 0.5 & 0.89 & 0.03 & 0.10 & -0.14 & 0.09 \\
\hline MOA-2012-BLG-410 & 8.9 & 3.6 & 4.2 & 0.99 & 0.10 & 0.15 & -0.14 & 0.07 \\
\hline OGLE-2012-BLG-0816 & 1.8 & 0.2 & 2.4 & 1.57 & 0.34 & 0.03 & -0.10 & 0.14 \\
\hline OGLE-2012-BLG-0211 & 9.9 & 2.9 & 2.9 & 0.99 & 0.08 & 0.10 & -0.03 & 0.08 \\
\hline MOA-2011-BLG-234 & 6.2 & 4.9 & 3.2 & 0.96 & 0.04 & 0.05 & -0.02 & 0.08 \\
\hline OGLE-2011-BLG-1105 & 3.1 & 2.6 & 6.9 & 0.93 & 0.06 & 0.07 & 0.00 & 0.17 \\
\hline MOA-2010-BLG-523 & 2.8 & 1.0 & 4.2 & 1.42 & 0.33 & 0.17 & 0.06 & 0.14 \\
\hline OGLE-2012-BLG-1274 & 8.5 & 1.0 & 1.1 & 1.03 & 0.04 & 0.04 & 0.07 & 0.04 \\
\hline OGLE-2012-BLG-0521 & 6.8 & 4.0 & 6.3 & 1.12 & 0.19 & 0.33 & 0.09 & 0.15 \\
\hline MOA-2011-BLG-034 & 12.4 & 3.2 & 2.2 & 0.94 & 0.05 & 0.09 & 0.11 & 0.15 \\
\hline MOA-2009-BLG-174 & 7.8 & 5.0 & 3.1 & 0.94 & 0.04 & 0.04 & 0.11 & 0.08 \\
\hline MOA-2009-BLG-456 & 9.0 & 1.9 & 2.0 & 1.00 & 0.06 & 0.06 & 0.13 & 0.10 \\
\hline MOA-2012-BLG-291 & 14.5 & 2.8 & 0.3 & 0.92 & 0.06 & 0.06 & 0.16 & 0.24 \\
\hline OGLE-2011-BLG-1410 & 3.9 & 1.7 & 9.5 & 1.35 & 0.41 & 0.23 & 0.22 & 0.37 \\
\hline MOA-2011-BLG-191 & 4.6 & 2.3 & 3.5 & 1.27 & 0.18 & 0.26 & 0.26 & 0.14 \\
\hline MOA-2011-BLG-445 & 4.8 & 2.6 & 8.5 & 1.26 & 0.32 & 0.31 & 0.26 & 0.31 \\
\hline OGLE-2007-BLG-514 & 6.6 & 2.7 & 3.1 & 1.09 & 0.11 & 0.16 & 0.29 & 0.23 \\
\hline OGLE-2011-BLG-0950 & 2.2 & 1.3 & 1.0 & 1.29 & 0.09 & 0.12 & 0.33 & 0.10 \\
\hline MOA-2009-BLG-259 & 2.2 & 0.2 & 1.7 & 1.59 & 0.24 & 0.01 & 0.34 & 0.19 \\
\hline MOA-2008-BLG-311 & 0.3 & 0.1 & 1.1 & 1.14 & 0.04 & 0.04 & 0.35 & 0.08 \\
\hline OGLE-2011-BLG-1072 & 5.7 & 2.2 & 2.5 & 1.20 & 0.12 & 0.17 & 0.36 & 0.12 \\
\hline MOA-2011-BLG-058 & 11.0 & 4.9 & 3.3 & 1.00 & 0.08 & 0.18 & 0.37 & 0.25 \\
\hline MOA-2008-BLG-310 & 6.2 & 1.7 & 1.8 & 1.09 & 0.06 & 0.07 & 0.41 & 0.11 \\
\hline MOA-2012-BLG-022 & 3.5 & 2.4 & 1.7 & 1.15 & 0.06 & 0.07 & 0.42 & 0.09 \\
\hline OGLE-2007-BLG-349 & 13.8 & 4.1 & 0.9 & 0.93 & 0.05 & 0.06 & 0.42 & 0.26 \\
\hline MOA-2006-BLG-099 & 0.6 & 0.3 & 3.6 & 1.08 & 0.07 & 0.06 & 0.44 & 0.21 \\
\hline OGLE-2006-BLG-265 & 9.4 & 2.6 & 2.8 & 1.00 & 0.06 & 0.07 & 0.46 & 0.19 \\
\hline OGLE-2012-BLG-0026 & 3.8 & 1.6 & 9.2 & 1.37 & 0.41 & 0.21 & 0.50 & 0.44 \\
\hline MOA-2010-BLG-311 & 3.9 & 1.7 & 2.9 & 1.33 & 0.19 & 0.24 & 0.51 & 0.19 \\
\hline MOA-2011-BLG-278 & 8.4 & 5.4 & 5.2 & 1.04 & 0.12 & 0.33 & 0.52 & 0.39 \\
\hline MOA-2010-BLG-037 & 3.8 & 1.6 & 1.7 & 1.31 & 0.14 & 0.23 & 0.55 & 0.20 \\
\hline
\end{tabular}

Notes. Column 1: star identifier; Col. 2: stellar age; Cols. 3 and 4: $1 \sigma$ lower and upper uncertainties for age estimates; Col. 5: stellar mass; Cols. 6 and 7: $1 \sigma$ lower and upper uncertainties for mass estimates; Cols. 8 and 9: [Fe/H] and its error from Bensby et al. (2013). 

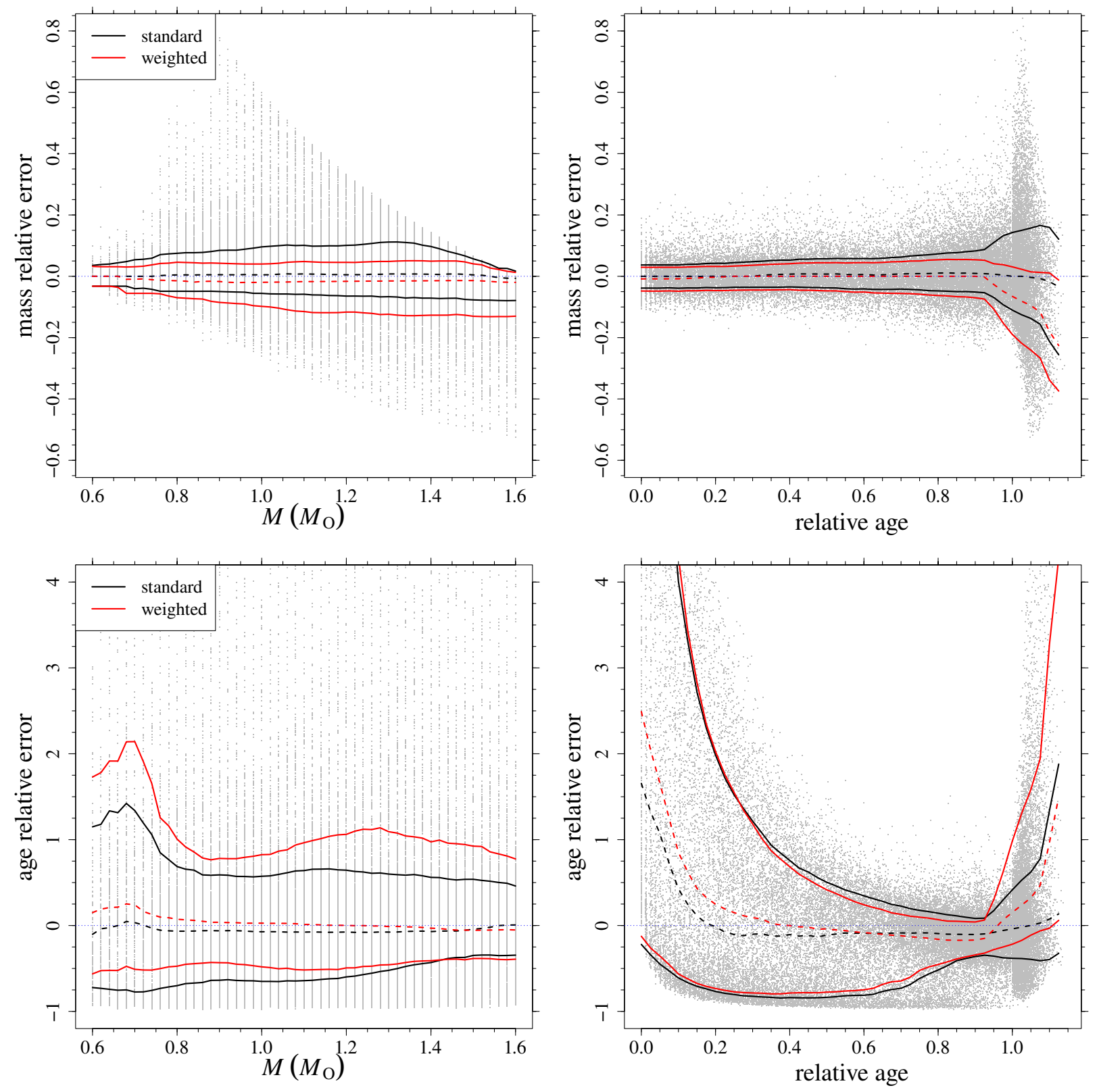

Fig. A.1. Top row: left Monte Carlo relative errors on mass estimate as a function of the true mass of the stars. The black lines show the position of the median (dashed line) and the $1 \sigma$ envelope (solid line). The red lines show the corresponding quantities, but for weighted estimates. Right: same as in the left panel, but as a function of the relative age of the stars. Bottom row: same as the top row, but for age estimates.

\section{Appendix A: Expected biases on grid estimates}

We present here an evaluation of the expected errors and possible bias of the technique adopted for age estimates. This information helps to evaluate the accuracy of the results presented in the paper.

For this test we sampled $N=50000$ artificial stars from the recovery grid. To mimic the microlensed sample characteristics, we restricted the sampling to stars up to the very first phase of RGB. The observational parameters of these stars are then subjected to a random Gaussian perturbation, assuming as observational errors $100 \mathrm{~K}$ in $T_{\text {eff }}, 0.2 \mathrm{dex}$ in $\log g$, and $0.1 \mathrm{dex}$ in $[\mathrm{Fe} / \mathrm{H}]$. The star masses and ages were then estimated by means of the SCEPtER technique.
The resulting relative errors in mass and age are presented in Fig. A.1, where a positive value in the relative error implies that the technique overestimates the considered parameter. The figure shows the trend of the relative errors versus the true mass of the star and its relative age ${ }^{3}$. To better follow the resulting trends in the figure we superimpose the median and the $1 \sigma$ error envelope, computed over a moving window (see Valle et al. 2015b, for details on the envelope computation). The figure shows some clear signatures of edge effects, such as the trend in the relative error of mass estimate versus the true mass (top left panel in Fig. A.1) or the huge distortion of age estimate at low relative age (bottom right panel in the figure), extensively discussed in

3 The relative age is defined as the ratio between the current age of the star and its age at the central hydrogen depletion. It is conventionally set to 0 at the ZAMS. 
Valle et al. (2014, 2015b). As an example, the trend in the top left panel of the figure at the high mass edge $\left(M=1.6 M_{\odot}\right)$ originates from the impossibility of obtaining a mass estimate above 1.6 $M_{\odot}$, the maximum value considered in the grid construction. Similar considerations apply to the other grid edges (Valle et al. 2015b). The tail toward age overestimation, visible at low masses in the bottom left panel, is due to the fact that for stars of mass lower than about $0.7 M_{\odot}$ the grid does not contain models at high relative age because of the constraint of the $15 \mathrm{Gyr}$ maximum age mentioned in Sect. 2.1. In other words for these masses the grid does not reach the later central hydrogen burning phases, which leads to more precise age estimates (see bottom right panel in Fig. A.1). The lack of these models causes the asymmetry in the envelope, typical of the first evolutionary phases. As expected (see e.g. Gai et al. 2011), mass and age estimates become more difficult in the subgiant phase (relative age higher than 1), since tracks are more closely packed in this grid zone. Moreover, for relative ages higher than about 1.1 a slight mass underestimation distortion appears, with a corresponding increase in age estimate (see the lower envelope boundary in the top right panel of Fig. A.1, and the corresponding upper boundary in the bottom right panel). The trend is due to the packing of the tracks toward the RGB ascension; low mass tracks pack more strictly than those of high mass, therefore the mass is more easily underestimated than overestimated.
For our purposes, the most interesting feature is the apparent lack of bias in the median of mass and age estimates visible in Fig. A.1 (with the exception of the first $20 \%$ of the evolution where edge effects dominate). It also appears from the bottom right panel of the figure that in the subgiant phase the age estimates present a tail toward overestimation.

It is often stated in the literature that mass and age estimates computed neglecting the evolutionary time step suffer from a bias and they could be improved by accounting for this correction (see e.g. Jørgensen \& Lindegren 2005; Casagrande et al. 2011). This statement also appears in Bensby et al. (2013) in relation to the microlensed star age estimates. However, this is not always true since the effect is strictly related to the employed technique and the considered evolutionary stages. In particular, for the results presented in this paper, the weighted estimates obtained adopting the evolutionary time step as weight are clearly biased toward age overestimation (bottom right panel in Fig. A.1). The effect is most evident in the subgiant phase, where the bias is higher than $100 \%$. In this phase, where tracks are closely crowded, the time step weight can make a large difference, leading to the selection of low-mass high-age models. Figure A.1 shows these trends by reporting the $1 \sigma$ envelope of the weighted estimates. Therefore, in the light of this result, in the present work we adopt unweighted estimates as a reference scenario. 